\title{
Fuzzy and Fourier Transforms
}

\author{
Irina Perfilieva, ${ }^{1}$ Petra Hod'áková ${ }^{2}$
}

${ }^{1}$ University of Ostrava, Inst. for Research and Applications of Fuzzy Modeling, Irina.Perfilieva@osu.cz
${ }^{2}$ University of Ostrava, Inst. for Research and Applications of Fuzzy Modeling, Petra.Hodakova@osu.cz

\begin{abstract}
The fuzzy transform ( $F$-transform for short) is a universal tool for a fuzzy modeling with convincing applications to image processing. The aim of this contribution is to explain the effect of the $F$ transform in image processing. With this purpose, we investigate properties of the Fourier transform over the $F$-transform components. We prove that the direct $F$-transform is a low-pass filter. This explains specific tools and methodologies that are developed in the $F$-transform applications to the image processing.
\end{abstract}

Keywords: Convolutions of functions, discrete $F$ transform, discrete Fourier transform

\section{Introduction}

Various kinds of transforms are used as powerful methods for solving many problems, including image processing. The main idea of them consists in transforming the original model into a special space where a computation is simpler. In this contribution, we will discuss the Fourier transform and the $F$-transform.

The Fourier transform is a well known method that is widely used in image processing. In general, we can say that the Fourier transform converts a function (image), considered in a time or spatial domain, into another function, considered in a frequency domain. In the case of images, the number of frequencies in a frequency domain is equal to the number of pixels in the image or spatial domain.

Transformation to a frequency domain is a very important tool in many applications. For example, applying filters to images in a frequency domain is computationally faster than doing the same in an image domain. Spectrum analysis is also widely used in speech analysis, image compression, search of periodicity in a wide variety of data in economics, biology, physics, etc.

In particular, the Fourier image analysis has several useful properties. For example, the operation of convolution in a spatial domain corresponds to the operation of multiplication in a frequency domain. This is important because multiplication is a simpler mathematical operation than convolution.

The $F$-transform is another technique discussed in this contribution. It performs a transformation of an original universe of functions into a universe of vectors. In more details, the $F$-transform establishes a correspondence between a set of continuous functions on an interval of real numbers and the set of n-dimensional (real) vectors.

The $F$-transform proves to be a successful methodology with various applications in image compression and reconstruction $([4],[5])$, image fusion ([2], [3]), numeric solution of differential equations $([7])$, time-series procession $([6])$. It turned out that the $F$-transform is very general and as powerful in many applications as conventional transforms. Moreover, sometimes the $F$-transform can be more efficient than its counterparts.

The structure of this paper is as following: Section 2 introduces notions of a fuzzy partition and a generating function of an $h$-uniform fuzzy partition. In this section, the direct form of a discrete $F$-transform is reminded and its representation in the form of a convolution is introduced. In Section 3 , the properties of a convolution are recalled. Section 4 reminds a definition of the discrete Fourier transform. In Section 5, an application of the discrete Fourier transform to the $F$-transform components is discussed. Section 6 presents examples, and Section 7 concludes the contribution.

\section{F-transform as Convolution}

In this section, we aim at expressing the $F$ transform in a form of a convolution of two functions. We will start with reminding basic definitions regarding the $F$-transform. We will focus on the discrete $F$-transform only.

\subsection{Discrete $F$-transform}

Let us consider the discrete $F$-transform [1]. We choose an interval $[a, b]$ as a universe, and assume that a function $f$ is given at points $p_{0}, \ldots, p_{l-1} \in$ $[a, b]$.

Below, we recall the definition of a fuzzy partition. Let $a=x_{0}<\cdots<x_{n}=b, n \geq 3$ be fixed nodes within $[a, b]$. Fuzzy sets $A_{1}, \ldots, A_{n-1}$ identified with their membership functions $A_{1}, \ldots, A_{n-1}$, defined on $[a, b]$, establish a fuzzy partition of $[a, b]$ if they fulfill the following conditions for $k=$ $1, \ldots, n-1$ :

1) $A_{k}:[a, b] \rightarrow[0,1], A_{k}\left(x_{k}\right)=1$;

2) $A_{k}(x)=0$ if $x \notin\left(x_{k-1}, x_{k+1}\right), k=1, \ldots, n-1$;

3) $A_{k}(x)$ is continuous; 
4) $A_{k}(x)$ strictly increase on $\left[x_{k-1}, x_{k}\right]$,

$k=1, \ldots, n-1$; and strictly decrease on $\left[x_{k}, x_{k+1}\right], k=1, \ldots, n-1$

5) $\sum_{k=1}^{n} A_{k}(x)=1, x \in\left[x_{1}, x_{n-1}\right]$.

$A_{1}, \ldots, A_{n-1}$ are called basic functions.

We say that the fuzzy partition given by $A_{1}, \ldots, A_{n-1}$, is an $h$-uniform fuzzy partition if the nodes $x_{k}=a+h k, k=0, \ldots, n$, are equidistant, $h=(b-a) / n$ and two additional properties are met:

6) $A_{k}\left(x_{k}-x\right)=A_{k}\left(x_{k}+x\right), x \in[0, h], k=$ $1, \ldots, n-1$;

7) $A_{k}(x)=A_{k-1}(x-h), k=2, \ldots, n-1, x \in$ $\left[x_{k-1}, x_{k+1}\right]$.

Assume that fuzzy sets $A_{1}, \ldots, A_{n-1}$ establish a fuzzy partition of $[a, b]$ and $f: P \longrightarrow \mathbb{R}$ is a discrete real valued function defined on the set $P=\left\{p_{0}, \ldots, p_{l-1}\right\}$ where $P \subseteq[a, b]$ and $l>$ $n$. The following vector of real numbers $\mathbf{F}_{n}[f]=$ $\left[F_{1}, \ldots, F_{n-1}\right]$ is the (direct) discrete $F$-transform of $f$ w.r.t. $A_{1}, \ldots, A_{n-1}$ where the $k$-th component $F_{k}$ is defined by

$$
F_{k}=\frac{\sum_{j=0}^{l-1} A_{k}\left(p_{j}\right) f\left(p_{j}\right)}{\sum_{j=0}^{l-1} A_{k}\left(p_{j}\right)}, k=1, \ldots, n-1 .
$$

By using an inversion formula we can approximately reconstruct function $f$ from the vector of components of its direct discrete $F$-transform. We define [1] the inverse discrete F-transform as

$$
f_{F, n}\left(p_{j}\right)=\sum_{k=1}^{n-1} F_{k} A_{k}\left(p_{j}\right), j=0, \ldots, l-1 .
$$

Moreover, the following Theorem 1 says that the inverse discrete F-transform $f_{F, n}$ can approximate the original function $f$ at common nodes with an arbitrary precision (proved in [1]).

\section{Theorem 1}

Let a function $f$ be given at nodes $p_{0}, \ldots, p_{l-1}$ constituting the set $P \subseteq[a, b]$. Then, for any $\varepsilon>0$, there exist $n_{\varepsilon}$ and a fuzzy partition $A_{1}, \ldots, A_{n_{\varepsilon}}$ of $[a, b]$ such that $P$ is sufficiently dense with respect to $A_{1}, \ldots, A_{n_{\varepsilon}}$ and for all $p \in\left\{p_{0}, \ldots, p_{l-1}\right\}$

$$
\left|f(p)-f_{F, n_{\varepsilon}}(p)\right|<\varepsilon
$$

holds true.

\subsection{F-Transform as Convolution}

Let us assume that the interval $[a, b]$ is $h$-uniformly partitioned by fuzzy sets $A_{1}, \ldots, A_{n-1}, f$ is a discrete function, and the $F$-transform of a discrete function $f$ is given by $\mathbf{F}_{n}[f]$ with components obtained by $(1)$.

It is easy to see that if the fuzzy partition $A_{1}, \ldots, A_{n-1}$ of $[a, b]$ is $h$-uniform, then there exists an even function

$$
A:[-h, h] \longrightarrow[0,1]
$$

such that for all $k=1, \ldots, n-1$,

$$
A_{k}(x)=A\left(x-x_{k}\right)=A\left(x_{k}-x\right), x \in\left[x_{k-1}, x_{k+1}\right] .
$$

We call $A$ a generating function of an $h$-uniform fuzzy partition.

The example of a triangular generating function $A$ and the respective $h$-uniform fuzzy partition $A_{1}, \ldots, A_{n-1}$ is given in Figure 1.

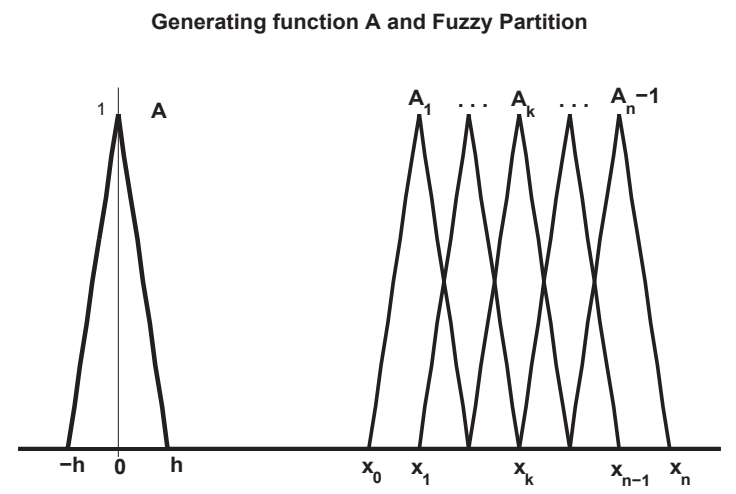

Figure 1: Generating function $A$ of an $h$-uniform fuzzy partition.

Let us assume that points $p_{0}, \ldots, p_{l-1}$ are equidistant in the interval $[a, b]$ and moreover $p_{j}=a+$ $j h / m ; j=0, \ldots, l-1$, where $m$ and $l$ are connected by the following equality: $l=n m+1$. Thus chosen points $p_{0}, \ldots, p_{l-1}$ assure that the nodes $x_{0}, \ldots, x_{n}$ are among them, i.e. for each $k=0, \ldots, n$, there exists $j$ such that $x_{k}=p_{j}$. Moreover, the following Lemma 1 holds true.

\section{Lemma 1}

Let $A_{1}, \ldots, A_{n-1}$ establish an $h$-uniform fuzzy partition of $[a, b]$ and points $p_{0}, \ldots, p_{l-1}$ from $[a, b]$ are chosen as above. Then there exists a constant $c>0$ such that for all $k=1, \ldots, n-1$,

$$
\sum_{j=0}^{l-1} A_{k}\left(p_{j}\right)=c .
$$

PROOF: In order to prove (2), it is sufficient to show that for all $k=1, \ldots, n-2$,

$$
\sum_{j=0}^{l-1} A_{k+1}\left(p_{j}\right)=\sum_{j=0}^{l-1} A_{k}\left(p_{j}\right) .
$$

Indeed, the uniformity of our partition and the fact that

$$
A_{k+1}\left(p_{j+m}\right)=A_{k}\left(p_{j}\right), j=0, \ldots, l-1-m,
$$

leads to

$$
\begin{array}{r}
\sum_{j=0}^{l-1} A_{k+1}\left(p_{j}\right)=A_{k+1}\left(p_{k m}\right)+\cdots+A_{k+1}\left(p_{(k+2) m}\right)= \\
A_{k}\left(p_{(k-1) m}\right)+\cdots+A_{k}\left(p_{(k+1) m}\right)=\sum_{j=0}^{l-1} A_{k}\left(p_{j}\right), \\
k=1, \ldots, n-2 .
\end{array}
$$




\section{Remark 1}

Let us remark that (2) is not the generalized Ruspini condition, because the sum is taken over points $p_{0}, \ldots, p_{l-1}$. Actually, the sum in (2) is taken over those points that are covered by a single basic function $A_{k}, k=1, \ldots, n-1$.

By (2), the expression (1) can be rewritten as follows:

$$
F_{k}=\frac{\sum_{j=0}^{l-1} A\left(x_{k}-p_{j}\right) f\left(p_{j}\right)}{c} ; k=1, \ldots, n-1 .
$$

Let us consider $F_{k}$ as a value of a discrete function $F$, defined on the set $\mathbf{Z}_{n-1}=\{1, \ldots, n-1\}$ with values from $\mathbb{R}$ such that $F: \mathbf{Z}_{n-1} \longrightarrow \mathbb{R}$ and $F(k)=$ $F_{k}$. We will use (4) for an analytic extension of $F$ from $\mathbf{Z}_{n-1}$ to $\mathbf{Z}_{l}=\{0,1, \ldots, l-1\}$, so that

$$
F(t)=\frac{\sum_{j=0}^{l-1} A\left(p_{t}-p_{j}\right) f\left(p_{j}\right)}{c} ; t=0, \ldots, l-1 .
$$

Similarly, we can assume that functions $A$ and $f$ are defined on the set $\mathbf{Z}_{l}$ and rewrite (5) into

$$
F(t)=\frac{\sum_{j=0}^{l-1} A(t-j) f(j)}{c} ; t=0, \ldots, l-1 .
$$

Finally, we will normalize values of $A$ dividing them by $c$ and keep the same denotation $A$ for the normalized function. Then without loss of generality, we will continue working with the below given expression for $F$ :

$$
F(t)=\sum_{j=0}^{l-1} A(t-j) f(j) ; t=0, \ldots, l-1 .
$$

Analyzing (7), we see that the function $F: \mathbf{Z}_{l} \longrightarrow$ $\mathbb{R}$ is a convolution (see e.g., [8], [9]) of two discrete functions $A$ and $f$. Let us remark that $F$ contains the $F$-transform components $F_{k}, k=1, \ldots, n-1$ among its values.

\section{Convolution of Functions}

Let us briefly remind the general definition of a convolution of functions (see e.g., [8]) and its properties. Let two functions $h, g: \mathbf{Z}_{l} \longrightarrow \mathbf{Z}_{l}$ be defined on the set of natural numbers $\mathbf{Z}_{l}=\{0,1, \ldots, l-1\}$. Then a discrete convolution $h * g$ is a function $h * g: \mathbf{Z}_{l} \longrightarrow \mathbf{Z}_{l}$ defined by

$$
(h * g)(t)=\sum_{j=0}^{l-1} h(t-j) g(j) .
$$

The important property is that the (discrete) Fourier transform (see below) of a convolution of functions is the product of their Fourier transforms, i.e.

$$
\widehat{h * g}=\hat{h} \cdot \hat{g},
$$

where symbols $\widehat{h * g}, \hat{h}, \hat{g}$ denote the Fourier transforms of $h * g, h, g$, respectively.

\section{Discrete Fourier Transform}

In this section, we recall the definition of the discrete Fourier transform (see e.g., [8]) as well as some properties which will be used further on. Let $h: \mathbf{Z}_{l} \longrightarrow \mathbf{C}$ be a function from the set $\mathbf{Z}_{l}=$ $\{0,1, \ldots, l-1\}$ to the set of complex numbers $\mathbf{C}$. Then the discrete Fourier transform $\hat{h}: \mathbf{Z}_{l} \longrightarrow \mathbf{C}$ of $h$ has the following representation:

$$
\hat{h}(u)=\sum_{t=0}^{l-1} h(t) \cdot \exp (-2 \pi i t u / l) ; u \in \mathbf{Z}_{l} .
$$

The inversion formula recovers the function $h$ from its discrete Fourier transform $\hat{h}$. It is defined by

$$
h(t)=\frac{1}{l} \sum_{u=0}^{l-1} \hat{h}(u) \cdot \exp (2 \pi i t u / l) ; t \in \mathbf{Z}_{l} .
$$

\section{Discrete Fourier Transform of $F$-transform Components}

Let the function $F: \mathbf{Z}_{l} \longrightarrow \mathbb{R}$ be given by (7) and coincide with the $F$-transform components at certain nodes. The discrete Fourier transform of $F$ is equal to:

$$
\hat{F}(u)=\sum_{t=0}^{l-1} F(t) \cdot \exp (-2 \pi i t u / l) ; u=0, \ldots, l-1 .
$$

Using the inversion formula of the Fourier transform we will obtain the following representation of the function $F$ :

$$
F(t)=\frac{1}{l} \sum_{u=0}^{l-1} \hat{F}(u) \cdot \exp (2 \pi i t u / l) ; t=0, \ldots, l-1,
$$

where expressions

$$
\exp (2 \pi i t u / l), u=0, \ldots, l-1
$$

represent basis functions of the Fourier decomposition (11).

Our purpose is to estimate values of $\hat{F}(u)$ for each frequency $u, u=0, \ldots, l-1$.

\section{Main Result}

\section{Theorem 2}

Let $\hat{f}: \mathbf{Z}_{l} \longrightarrow \mathbb{R}$ be the Fourier transform of a function $f: \mathbf{Z}_{l} \longrightarrow \mathbb{R}$. Let $n \geq 3$ and $A_{1}, \ldots, A_{n-1}$ be an $h$-uniform fuzzy partition of $[a, b]$ where $h=$ $\frac{b-a}{n}$. Assume that the fuzzy partition $A_{1}, \ldots, A_{n-1}$ has $A:[-h, h] \longrightarrow[0,1]$ as a generating function and moreover, $A$ is of a triangular shape, i.e. $A(x)$ is defined on $[-h, h]$ by

$$
A(x)= \begin{cases}1-\frac{|x|}{h}, & |x| \leq h, \\ 0, & |x|>h .\end{cases}
$$


Let $F: \mathbf{Z}_{l} \longrightarrow \mathbb{R}$ be the discrete function given by (7), which contains the F-transform components of $f$ among its values. Then the Fourier transform of $F$ is given by

$$
\begin{aligned}
\hat{F}(0) & =\hat{f}(0) \\
\hat{F}(u) & \approx \frac{m n^{2}}{2 \pi^{2} u^{2}} \exp (-2 \pi i u / n)\left(1-\cos \frac{2 \pi u}{n}\right) \cdot \hat{f}(u) \\
u & =1, \ldots, l-1
\end{aligned}
$$

where $m$ is a fixed parameter.

PROOF: Let us consider $F$ in the form of the convolution (7). Using the property (8), we can write

$$
\hat{F}(u)=\hat{A}(u) \cdot \hat{f}(u) \text {. }
$$

Now we will estimate $\hat{A}(u)$ and leave $\hat{f}(u)$ as it is. Recall that in (14), the function $A$ is normalized. We use the general expression (9) to compute $\hat{A}(u)$ :

$$
\hat{A}(u)=\sum_{t=0}^{l-1} A(t) \cdot \exp (-2 \pi i t u / l) ; u=0, \ldots, l-1
$$

In particular, $A(0)=1$, which easily follows from normalization of $A$. For other values $u=1, \ldots, l-$ 1, the expression above will be approximated by respective integrals, so that

$$
\begin{gathered}
\hat{A}(u) \approx \frac{m}{h} \exp (-2 \pi i u / n) \int_{-h}^{h} A(x) \cos \frac{2 \pi x u}{n h} d x- \\
i \frac{m}{h} \exp (-2 \pi i u / n) \int_{-h}^{h} A(x) \sin \frac{2 \pi x u}{n h} d x \\
u=1, \ldots, l-1 .
\end{gathered}
$$

Because $A$ is an even function on $[-h, h]$ (cf. (13)), the second integral in the expression above is 0 . By direct integration of $\int_{-h}^{h} A(x) \cos \frac{2 \pi x u}{n h} d x$, we obtain the following approximate values of $\hat{A}(u), u=$ $1, \ldots, l-1$ :

$$
\hat{A}(u) \approx \frac{m n^{2}}{2 \pi^{2} u^{2}} \exp (-2 \pi i u / n)\left(1-\cos \frac{2 \pi u}{n}\right) .
$$

Substitution of (15) into (14) gives us the desired expression:

$$
\begin{aligned}
\hat{F}(u) & \approx \frac{m n^{2}}{2 \pi^{2} u^{2}} \exp (-2 \pi i u / n)\left(1-\cos \frac{2 \pi u}{n}\right) \cdot \hat{f}(u) \\
& u=1, \ldots, l-1
\end{aligned}
$$

\section{Corollary 1}

Let the assumptions of the Theorem 2 be fulfilled. Then the influence of the Fourier coefficient $\hat{f}(u)$ in the representation (11) is weakened by the factor $\frac{1}{u^{2}}$.

In other words, Corollary 1 states that every $F$ transform component works as a low-pass filter of an original function.

\section{Graphical Example}

Below, we illustrate the idea described above on a particular example. We take an interval $[0,2 \pi]$ as a universe and two discrete functions $\sin x, \sin 5 x$, both defined at points $0=p_{0}, \ldots, p_{80}=2 \pi$, where $p_{j}=\frac{j \pi}{40}, j=0, \ldots, 80$. We form a $h$-uniform fuzzy partition of the interval $[0,2 \pi]$ represented by triangular basic functions $A_{1}, \ldots, A_{7}$ over the nodes $x_{0}, \ldots, x_{8}$, where the distance between each two neighboring nodes $h=\frac{\pi}{4}$.

For both functions we compute the direct discrete $F$-transform and the inverse discrete $F$-transform with respect to the given fuzzy partition of the interval $[0,2 \pi]$. The function $\sin x$ with its inverse $F$-transform and the $F$-transform components is depicted on Figure 2 and the function $\sin 5 x$ with the corresponding inverse $F$-transform and the $F$ transform components is shown on Figure 3. Both functions and their $F$-transforms are represented at points $p_{j}, j=0, \ldots, 80$, although graphs seem to be continuous.

It is easy to see that the oscillation of $\sin 5 x$ is higher than that of $\sin x$. Therefore by Lemma 2 from [1], for the same partition, the approximation of $\sin x$ by its inverse $F$-transform is closer to the original function than the approximation of $\sin 5 x$ by its inverse $F$-transform.

In the frequency domain of the Fourier spectra, peaks of a high oscillating function give evidence of a presence of high frequencies. As can be seen from Figure 3, the $F$-transform components of $\sin 5 x$ reduce the influence of high frequencies in the respective approximation given by the inverse $F$-transform.

Therefore, in order to increase the quality of approximation of a high oscillating function by its inverse F-transform it is necessary to increase the value of $n$ leaving all other parameters unchanged, as can be seen on Figure 4. However, this requires a thorough analysis of the expression (15).

\section{Conclusion}

Our investigation was focused on the discrete $F$ transform and its effect in image processing. After a brief introduction, the discrete $F$-transform was presented in the form of a convolution. We investigated properties of the discrete Fourier transform of the direct discrete $F$-transform. We proved that every $F$-transform component works as a low-pass filter of an original function.

\section{Acknowledgement}

The research was partially supported by the projects 1M0572 of MŠMT ČR (DAR) and SGS7/PRF/2011 (Soft Computing Methods in Particular problems of Image Processing. 


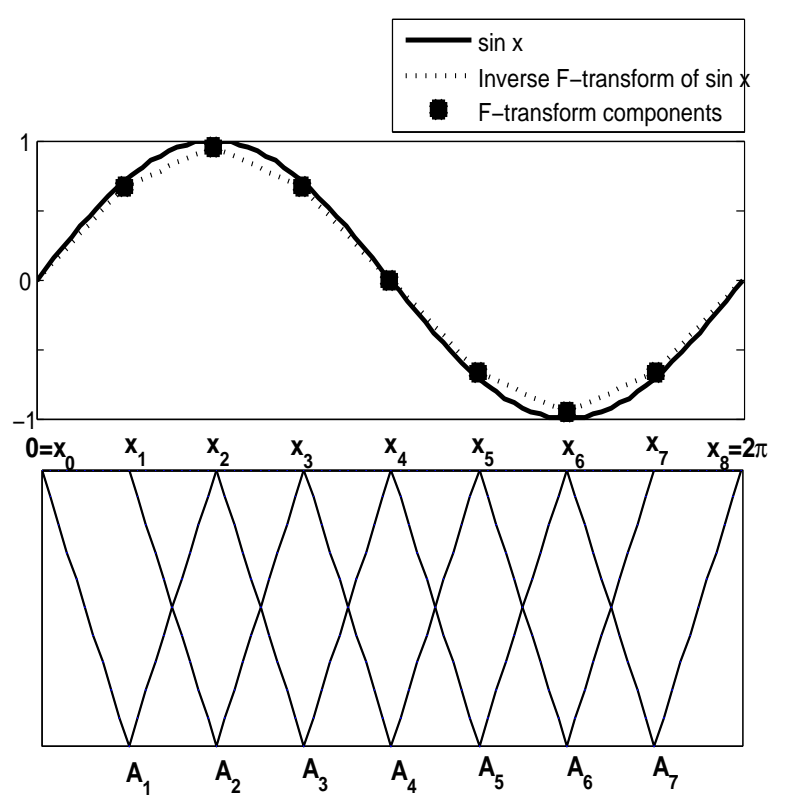

Figure 2: Above: Function $\sin x$, its inverse $F$ transform and corresponding 7 components of direct F-transform; Below: Fuzzy partition of $[0,2 \pi]$.

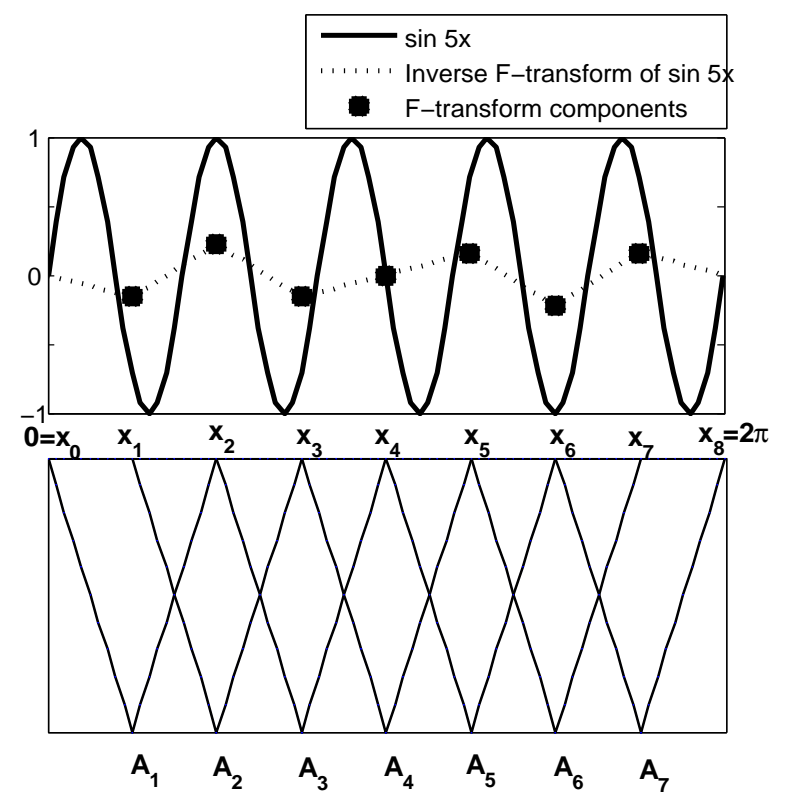

Figure 3: Above: Function $\sin (5 x)$, its inverse $F$ transform and corresponding 7 components of direct F-transform; Below: Fuzzy partition of $[0,2 \pi]$.

\section{References}

[1] I. Perfilieva, Fuzzy Transforms: Theory and applications. Fuzzy Sets and Systems, Vol. 157, pp. 993-1023, Elsevier, 2006.

[2] I. Perfilieva and M. Daňková: Image fusion on the basis of fuzzy transforms. Proc. $8^{\text {th }}$ Int. FLINS Conf., Madrid, pp. 471-476, 2008.

[3] I. Perfilieva, M. Daňková, P. Hod'áková and M. Vajgl: F-Transform Based Image Fusion. InTech, 2010.

[4] I. Perfilieva, V. Pavliska, M. Vajgl and B. De

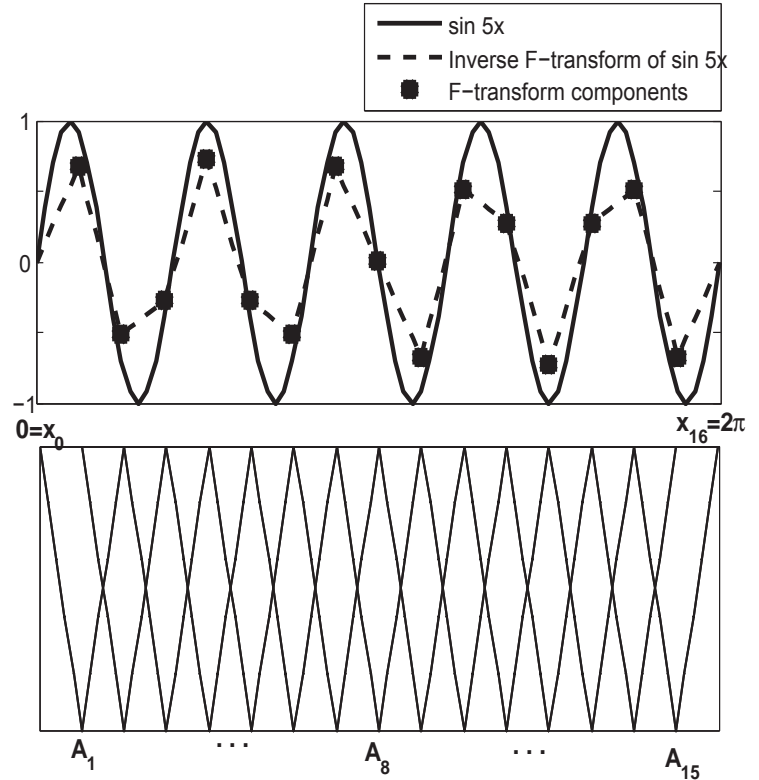

Figure 4: Above: Function $\sin (5 x)$, its inverse $F$ transform and corresponding 15 components of direct F-transform; Below: Fuzzy partition of $[0,2 \pi]$.

Baets: Advanced image compression on the basis of fuzzy transforms. Proc. Conf. IPMU'2008, pp. 1167-1174, Torremolinos (Malaga), Spain, 2008.

[5] F. Di Martino, V. Loia, I. Perfilieva, S. Sessa: An image coding/decoding method based on direct and inverse fuzzy transforms. International Journal of Appr. reasoning, Vol. 48, pp. 110131, 2008.

[6] I. Perfilieva, V. Novák, V. Pavliska, A. Dvořák and M. Štěpnička: Analysis and prediction of time series using fuzzy transform. Proc. of WCCI 2008, IEEE Int. Conf. on Neural Networks, pp. 3875-3879, Hong Kong, 2008.

[7] M. Štěpnička and R. Valášek: Numerical solution of partial differential equations with help of fuzzy transform. Proc. of the 2005 IEEE International Conference on Fuzzy Systems (FUZZIEEE'05), pp. 1153-1162, Reno, Nevada, 2005.

[8] G. Bachman, L. Narici and E. Beckenstein. Fourier and Wavelet Analysis, Springer-Verlag, New York, 2000.

[9] I. Perfilieva and V. Kreinovich, Towards a General Description of Translation-Invariant and Translation-Covariant Linear Transformations: A Natural Justification of Fourier Transforms and Fuzzy Transforms. Proc. of the IFSAAFSS'2011 Congress, Surabaya and Bali Island, INDONESIA, to appear. 